\title{
Incidence and Factors of Hypoglycemia in Perioperative Patients with Type 2 Diabetes Mellitus on Insulin Pump Therapy
}

\author{
Xiaohua Lu ${ }^{1, \dagger}$, Youshuang $\mathrm{Zhu}^{2, \dagger}$, Wane Zhao', Yan Liu ${ }^{1}$, Xixi Luo ${ }^{1}$, Yan Zhou ${ }^{1, *}$ \\ ${ }^{1}$ Department of Endocrinology, First Affiliated Hospital of Jinan University, Guangzhou, China \\ ${ }^{2}$ Department of Burn and Plastic Surgery and Intervention, First Affiliated Hospital of Jinan University, Guangzhou, China \\ Email address: \\ 992054881@qq.com (Yan Zhou) \\ ${ }^{*}$ Corresponding author \\ $\dagger$ Xiaohua $\mathrm{Lu}$ and Youshuang Zhu are co-first authors.
}

\section{To cite this article:}

Xiaohua Lu, Youshuang Zhu, Wane Zhao, Yan Liu, Xixi Luo, Yan Zhou. Incidence and Factors of Hypoglycemia in Perioperative Patients with Type 2 Diabetes Mellitus on Insulin Pump Therapy. American Journal of Nursing Science. Vol. 10, No. 2, 2021 , pp. $128-132$. doi: 10.11648/j.ajns.20211002.13

Received: February 16, 2021; Accepted: March 13, 2021; Published: March 26, 2021

\begin{abstract}
Objective: To estimate the incidence of and identified factors associated with hypoglycemia among perioperative diabetes patients with insulin pump therapy. Methods: This was a retrospective cohort study of type 2 diabetes patients with insulin pump therapy newly received surgery. All patients were divided into hypoglycemia group and non-hypoglycemia group according to whether they experienced hypoglycemia. Information about clinical characteristics and treatments were collected and compared between two groups. We estimated the incidence of hypoglycemia, and identified factors associated with hypoglycemia by using multivariable logistic regression. Results: A total of 172 episodes of hypoglycemia were observed in 88 (39.8\%) patients, among which, $146(84.9 \%)$ were level 1 hypoglycemia $(<3.9 \mathrm{mmol} / \mathrm{L})$ and $26(15.1 \%)$ were level $2(<3.0$ $\mathrm{mmol} / \mathrm{L}$ ). No severe hypoglycemia (level 3) was reported in this study. Results from the multivariable logistic regression model found that diabetes patients with a longer duration of pump use were more likely to have hypoglycemia (AOR=1.09 with $95 \%$ $\mathrm{CI}=1.04-1.15$ ). However, those diabetes patients who were with higher BMI and eGFR were less likely to have hypoglycemia ( $\mathrm{AOR}=0.86$ with $95 \% \mathrm{CI}=0.76-0.98 ; \mathrm{AOR}=0.86$ with $95 \% \mathrm{CI}=0.76-0.98$, respectively). Conclusion: Perioperative diabetes patients with insulin pump therapy were prone to develop mild to moderate hypoglycemia. The long-term of receiving insulin pump therapy during the perioperative period, with lower BMI and eGFR, put type 2 diabetes at higher risks in hypoglycemia. It may help healthcare providers to screen hypoglycemia among type 2 diabetes patients with receiving long-term insulin pump therapy.
\end{abstract}

Keywords: Continuous Subcutaneous Insulin Infusion, Insulin Pump Therapy, Perioperative Period, Type 2 Diabetes Mellitus, Hypoglycemia

\section{Introduction}

Continuous subcutaneous insulin infusion (CSII) therapy, also known as insulin pump therapy, has been identified as an optimal infusion method that simulates endogenous insulin secretion to a large extent [1]. According to China Insulin Pump Clinical Guideline (2014), using short-term insulin pump therapy has been indicated to benefits for perioperative glycemic control in diabetic patients [2]. The benefits of using CSII in the perioperative period may include a significant reduction in the risks of hyperglycemic crises, hyperglycemia, and mortality [3, 4] However, with the proliferation of insulin pump applications in the surgical setting, physicians will confront other challenges, especially the occurrence of hypoglycemia during the use of insulin pump $[5,6]$. Unmanaged hypoglycemia may increase the risks of comorbidities, especially neurological complications [7], impair surgical wound healing [8], may prolong ICU and hospital stay [9], and enhance mortality [7]. However, few studies reported risk factors for CSII-associated hypoglycemia 
in the perioperative period. As such, this study is aimed to investigate the incidence rate of hypoglycemia and its potential factors in perioperative diabetic patients managed by insulin pump therapy.

\section{Materials and Methods}

\subsection{Patients and Risk Factors}

This retrospective cohort study of type 2 diabetes was conducted in the surgery departments at the First Affiliated Hospital of Jinan University. The target population was limited to adults (aged $\geq 18$ ) with concurrent type 2 diabetes patients with insulin pump therapy. For this study, individuals who were diagnosed with pregnancy diabetes were excluded from the analysis. We also excluded individuals who had severe infections, acute metabolic complications (including diabetic ketoacidosis and hyperosmolar hyperglycemic syndrome), cardiopulmonary insufficiency, and severe liver and kidney dysfunction. The final study sample size included 221 adults with type 2 diabetes. The study was performed in accordance with the Declaration of Helsinki and the Ethical Guidelines for Clinical Research. The Research Ethical Committee approved the research protocol of the First Affiliated Hospital of Jinan University (ref: [2017]029).

Hypoglycemia was measured as a categorical variable during perioperative periods based on the results of the fingerstick glucose test. The International Hypoglycemia Study Group (IHSG) position statement proposes a glucose concentration of $3.9 \mathrm{mmol} / \mathrm{L}(70 \mathrm{mg} / \mathrm{dl})$ or less be counted as hypoglycemia (level 1) albeit no need to be reported in clinical studies. A level of $<3.0 \mathrm{mmol} / \mathrm{L}(<54 \mathrm{mg} / \mathrm{dl})$ is sufficiently low to indicate serious, clinically important hypoglycemia (level 2), whether that level is associated with or without symptoms of hypoglycemia. Severe hypoglycemia (level 3), as defined by the American Diabetes Association, was glycemic value of $<2.8 \mathrm{mmol} / \mathrm{L}$ or manifests severe cognitive impairment requiring external assistance for recovery $[10,11]$. All participants were assigned to either the hypoglycemia group or the non-hypoglycemia group based on whether low glucose concertation $(<3.9 \mathrm{mmol} / \mathrm{L})$ was experienced.

The following covariates of prior to CSII therapy, hemoglobin A1c (HbAlc), serum creatinine $(\mathrm{Cr})$ and alanine transaminase (ALT) were examined after fasting for 8-10 hours. HbAlc, $\mathrm{Cr}$, and ALT were examined by high-performance liquid chromatography. The estimated glomerular filtrating rate (eGFR) was calculated using the Modification of Diet in Renal Disease (MDRD) Study equation. Diet details and symptoms of hypoglycemia were recorded once hypoglycemia had been experienced.

\subsection{Statistical Analysis}

First, we estimated the incidence of hypoglycemia events among adult type 2 diabetes patients. Then we compared variables between type 2 diabetes patients with and without hypoglycemia using chi-square tests for categorical variables and t-tests for continuous variables. Then we conducted a multivariable logistic regression model to identify factors associated with hypoglycemia among participants. The multivariable models included the variables of statistically significant in the previous descriptive analysis at $\mathrm{P}<0.05$. The parameter estimates from the models were presented as adjusted odds ratio (AOR) and their corresponding 95\% confidence intervals (CI). Sciences (SPSS) version 19.0 was used for statistical analysis.

\section{Results}

Incidence and characteristics of hypoglycemia in type 2 diabetes who used insulin pump in the perioperative period

Between July 2017 and December 2018, a total of 221 patients with type 2 diabetes who used insulin pump in the perioperative period were enrolled from surgery departments at the First Affiliated Hospital of Jinan University. Of the 221 participants, $88(39.8 \%)$ patients had hypoglycemia event at least one time, $98(44.3 \%)$ were male and $123(55.7 \%)$ were female. In our study, a total of 172 episodes of hypoglycemia were observed in $88(39.8 \%)$ patients; of whom, $58.0 \%(51 / 88)$ experienced hypoglycemic event only once, $26.1 \%(23 / 88)$ went through twice or three times and $15.9 \%(14 / 88)$ underwent more than three episodes of hypoglycemia. Among 88 patients with hypoglycemia, 34 patients $(38.6 \%)$ had level 1 hypoglycemia $(<3.9 \mathrm{mmol} / \mathrm{L}), 16(18.2 \%)$ patients had level $2(<3.0 \mathrm{mmol} / \mathrm{L})$, and 48 patients $(54.5 \%)$ had multiple levels of hypoglycemia. No severe hypoglycemia (level 3) was found in our study. Among 88 patients with hypoglycemia episodes, 24 patients $(27.3 \%)$ had hypoglycemia episodes in the preoperative period, 47 (53.4\%) patients got such episodes in the postoperative period, and 17 patients $(19.3 \%)$ had them both pre and post-operation periods. We also found that 52 (59.1\%) patients got hypoglycemia after fasting, which includes preoperative and postoperative fasting, getting a liquid diet, and having a poor appetite in the postoperative period (Table 1).

Table 1. The characteristics of hypoglycemia episodes among Chinese adults with type two diabetes in the perioperative period $(M \pm S D)$.

\begin{tabular}{ll}
\hline & Hypoglycemic group (n=88) \\
\hline Overall events (Mean, SD) & $172(1.95, \pm 0.89)$ \\
Number of Hypoglycemic episodes & $51(58.0 \%)$ \\
1 & $23(26.1 \%)$ \\
$2-3$ & $14(15.9 \%)$ \\
$\geq 4$ & \\
Level of Hypoglycemic episodes & $34(38.6 \%)$ \\
Level $1(<3.9 \mathrm{mmol} / \mathrm{L})$ & $16(18.2 \%)$ \\
Level $2(<3.0 \mathrm{mmol} / \mathrm{L})$ & $0(0 \%)$ \\
Level $3(<2.8 \mathrm{mmol} / \mathrm{L})$ & $48(54.5 \%)$ \\
Multiple levels & \\
Period of Hypoglycemic episodes & $24(27.3 \%)$ \\
Preoperative period & $47(53.4 \%)$ \\
Postoperative period & $17(19.3 \%)$ \\
Pre-post operation periods & \\
Before or after fasting & $36(40.9 \%)$ \\
Before & $52(59.1 \%)$ \\
After &
\end{tabular}

Clinical characteristics of type 2 diabetes who used insulin 
pump in the perioperative period

Our results showed that type 2 diabetes patients who were with longer duration of diabetes, with higher serum creatinine levels, with lower BMI, with lower eGFR, with the long-term use of CSII were more likely to have hypoglycemia than their counterparts (Table 2).

Mechanical problems and interruptions in insulin delivery included pump malfunction, power error, clogged or dropped infusion tubing, and insulin leakage from the tubing or reservoir. Twelve mechanical mistakes were recorded in the hypoglycemic group and 17 errors in the non-hypoglycemic group. Errors in pump use involved incorrect bolus insulin dosage (e.g. missing or reduced bolus insulin infusion). Nine errors in pump use were reported in the hypoglycemic group and ten errors in the non-hypoglycemic group. There were no significant differences in mechanical problems of insulin pump and errors in pump use between these two groups (Table 2).

Table 2. The clinical characteristics for Chinese adults with type 2 diabetes with and without hypoglycemia in the perioperative period $(M \pm S D)$.

\begin{tabular}{llll}
\hline & $\begin{array}{l}\text { Hypoglycemic } \\
\text { group (n=88) }\end{array}$ & $\begin{array}{l}\text { Non-hypoglycemic } \\
\text { group (n=133) }\end{array}$ & P-value \\
\hline Gender(n) & 0.41 & & \\
Male & 42 & 56 & \\
Female & 46 & 77 & 0.06 \\
Age & $64.63 \pm 11.73$ & $61.74 \pm 10.67$ & $0.03^{*}$ \\
Duration of Diabetes & $8.94 \pm 6.75$ & $7.02 \pm 6.04$ & $<0.01^{*}$ \\
(years) & $22.81 \pm 3.37$ & $24.44 \pm 3.76$ & 0.55 \\
BMI $(\mathrm{kg} / \mathrm{m} 2)$ & $8.80 \pm 2.30$ & $8.99 \pm 2.20$ & $0.02^{*}$ \\
HbA1c $(\%)$ & $101.32 \pm 84.70$ & $78.89 \pm 59.04$ & $0.01^{*}$ \\
Cr ( $\mu$ mmol/L) & $79.84 \pm 44.77$ & $93.74 \pm 35.98$ & 0.35 \\
eGFR (ml/min) & $26.27 \pm 35.50$ & $23.02 \pm 15.33$ & 0.38 \\
ALT (U/L) & $0.61 \pm 0.25$ & $0.59 \pm 0.21$ & 0.17 \\
Total insulin/kg (U/kg) & $0.30 \pm 0.12$ & $0.27 \pm 0.11$ & 0.78 \\
Basal insulin/kg (U/kg) & $0.32 \pm 0.18$ & $0.31 \pm 0.14$ & $<0.01^{*}$ \\
Bolus insulin/kg (U/kg) & 0.3214 & 0.79 \\
Duration of pump use & $14.57 \pm 9.33$ & $10.29 \pm 6.23$ & \\
(days) & & $17 \pm 1.04$ & \\
Mechanical problems & $12 \pm 1.17$ & & \\
\hline
\end{tabular}

*T test significant at $\mathrm{P}<0.05$

Risk factors for hypoglycemia in the perioperative period

Results from the multivariable logistic regression model found that diabetes patients with receiving insulin pump therapy who had higher BMI (Adjusted odds ratio $[\mathrm{AOR}]=0.86$ with 95\% CI=0.76-0.98), had higher eGFR (AOR=0.99 with $95 \% \mathrm{CI}=0.98-0.99$ ) were more likely to have hypoglycemia compared to their counterparts. However, those diabetes patients who had a longer duration of insulin pump use were more likely to have hypoglycemia compared to those who used a pump shortly ( $\mathrm{AOR}=1.09$ with $95 \% \mathrm{CI}=1.04-1.15)$ (Table 3$)$.

Table 3. Adjusted odds ratios of associations with hypoglycemia among Chinese adults with type two diabetes in the perioperative period.

\begin{tabular}{|c|c|c|c|c|c|}
\hline Variable $\dagger$ & B & SE & Wald $X^{2}$ & $P \ddagger$ & AOR 95\% CI \\
\hline BMI & -0.147 & 0.064 & 5.349 & 0.021 & $0.8630 .762-0.978$ \\
\hline eGFR & -0.011 & 0.005 & 5.985 & 0.014 & $0.9890 .980-0.998$ \\
\hline $\begin{array}{l}\text { Duration of pump } \\
\text { use }\end{array}$ & 0.087 & 0.026 & 11.077 & 0.001 & $1.091 \quad 1.037-1.149$ \\
\hline
\end{tabular}

\section{Discussion}

Diabetic patients need surgical treatment because of various complications or other reasons. Most of them live in non-endocrine departments. Hyperglycemia can increase infection rate and delay wound healing. In recent years, CSII is widely used by endocrinologists to control blood glucose in patients with hyperglycemia during perioperative period. Compared with daily multiple insulin infusion, CSII can better simulate physiological insulin secretion which reduce blood glucose fluctuation, shorten the time of blood glucose reaching the standard and reduce the occurrence of hypoglycemia, so as to control blood glucose safely and effectively. However, hypoglycemia is a common side effect in intensive insulin pump therapy. Severe hypoglycemia can cause cardiovascular events or all-cause mortality, especially in patients with cardiovascular risk factors; non-severe hypoglycemia does not increase cardiovascular events or all-cause mortality, so we must pay special attention to the occurrence of hypoglycemia during insulin pump treatment.

This study provides the first profile of the incidence rate of hypoglycemia in type 2 diabetes patients who received CSII therapy during the perioperative period. We found that about $39.8 \%$ of participants had at least one hypoglycemic episode. Although evidence indicated that using the insulin pump during the perioperative period may have benefits for patients, the incidence of hypoglycemia in our study was still much higher than the previous studies in general in-patients who received insulin treatments $[12,13]$. However, our results are still consistent with similar findings, according to which the incidence of CSII-associated hypoglycemia in perioperative settings was ranging from $2.8 \%$ to $50 \%$ based on different study designs [14, 15], specific populations, and the definitions of hypoglycemia.

Our study also found among the incidence of perioperative hypoglycemia, most of which were level 1 hypoglycemia $(<3.9 \mathrm{mmol} / \mathrm{L})$. Only $15.1 \%$ of patients experienced level 2 hypoglycemia $(<3.0 \mathrm{mmol} / \mathrm{L})$. No severe hypoglycemic episodes were reported in the study. The results of our studies were consistent with previous studies as well. Although the incidence rate of hypoglycemia among type 2 diabetes patients in the perioperative period, severe hypoglycemia is uncommon. The final goal of our study was to determine the factors associated with hypoglycemia among type 2 diabetes patients in the perioperative period. Our study only found BMI, eGFR, and duration of pump use as independent factors of CSII-associated hypoglycemia in the perioperative period. We found that type 2 diabetes patients who were with lower BMI, and with eGFR were more likely to have hypoglycemia. Our findings are consistent with previous studies focusing on the general type 2 diabetes population. Actually, in our study, the average BMI in the hypoglycemic group was $22.81 \mathrm{~kg} / \mathrm{m}^{2}$, falling in the healthy weight range. In contrast, the mean BMI in the non-hypoglycemic group was $24.44 \mathrm{~kg} / \mathrm{m}^{2}$. Previous studies indicated that the higher the BMI is, the greater the glycemic threshold for response to hypoglycemia is [16, 17]. Therefore, diabetes patients with higher BMI may be less 
likely to have hypoglycemia and may have milder subsequent symptoms of hypoglycemia. Moreover, higher BMI was considered as a protective factor for night-time hypoglycemia in patients with type 2 diabetes [18]. As such, tailoring insulin treatment for perioperative patients should take BMI into account.

We also found patients with lower eGFR were more likely to have hypoglycemia. Lower eGFR may directly reflect patients with declined renal function that is commonly associated with the occurrence of hypoglycemia. Therefore, hypoglycemic events are more likely to occur in patients with impaired renal function during insulin pump treatment[19]. Therefore, it is important for physicians to be aware of adjusting insulin dosage based on patient's eGFR or renal function, monitor closely, and investigate its potential risk of hypoglycemia in perioperative management.

Finally, our results indicated that the longer the insulin pump use, the higher the risk of hypoglycemia presents. In that case, Liu et al proposed a steady decline of total daily insulin dose, a proximately $24-35 \%$ reduction at the end of two-week therapy, in patients with type 2 diabetes treated with CSII [20]. Actually, previous studies have shown that patients with longer duration of insulin therapy are more likely to have risk factors of hypoglycemia [21, 22].

A number of limitations and strengths of our study should be noted. First, an observational study may introduce selection bias or unmeasurable confounding factors. Second, our scope was limited to one hospital; therefore, our results may not be generalized to other hospitals or general populations. The future study may focus on multiple institutions and larger sample sizes to be more generalized. Third, with respect to diabetes severity, our study didn't include any item that probes the extent to these factors affect the risk of hypoglycemia. However, the eGFR and duration of diabetes may indirectly reflect the severity of diabetes.

\section{Conclusion}

In conclusion, our findings provide the first estimates of the incidence rate of hypoglycemia in diabetes patients who received CSII therapy during the perioperative period. Perioperative diabetes patients were prone to develop mild to moderate hypoglycemia, especially in postoperative period. No severe hypoglycemic episodes happened, which means CSII therapy has a good safety in the treatment of the perioperative diabetes patients in non-endocrinology department. We found lower likelihood of having hypoglycemia associated with higher BMI and eGFR among adult type 2 diabetes. However, an association between longer insulin pump use and having a hypoglycemia event was observed in our study. To avoid hypoglycemia, practitioners should pay attention to the patients' BMI and eGFR, increase the frequency of blood glucose monitoring, adjust insulin dose appropriately during the perioperative period especially in those with long-term receiving insulin therapy.

\section{Author Contribution}

$\mathrm{Lu} \mathrm{XH}$ and Zhu YS were responsible for the study conception, design and the manuscript drafting. Liu $\mathrm{Y}$ and Luo XX were responsible for the data acquisition and analysis. Zhou Y were responsible for the manuscript critical revision.

\section{Acknowledgements}

The work was supported by grants from Guangdong Provincial Medical Research Fund Project in 2019 (No. B2019012) and Guangdong Provincial Medical Research Fund Project in 2020 (No. A2020574).

\section{References}

[1] Boyle ME, Seifert KM, Beer KA, Apsey HA, Nassar AA, Littman SD, et al. Guidelines for application of continuous subcutaneous insulin infusion (insulin pump) therapy in the perioperative period. Journal of diabetes science and technology. 2012; 6 (1): 184-190.

[2] $\mathrm{Mu}$ Y, Yin S, Chinese Medical Practitioners Association, Endocrinology and Metabolism Branch, Chinese Society of Endocrinology, Chinese Diabetes Society. Insulin pump therapy guidelines for China (July 2010). Journal of diabetes. 2012; 4 (2): 127-139.

[3] Leung V, Ragbir-Toolsie K. Perioperative management of patients with diabetes. Health services insights. 2017; 10: 1-5.

[4] Kotagal M, Symons RG, Hirsch IB, Umpierrez GE, Dellinger EP, Farrokhi ET, et al. Perioperative hyperglycemia and risk of adverse events among patients with and without diabetes. Annals of surgery. 2015; 261 (1): 97-103.

[5] Reis J, Pignatelli D, Madeira D, Castedo JL, de Oliveira MC. Subcutaneous insulin pump therapy in a diabetic patient submitted to major surgery. Postgraduate medical journal. 1988; 64 (750): 335-338.

[6] White W, Montalvo H, Monday JM. Continuous subcutaneous insulin infusion during general anesthesia: a case report. AANA journal. 2004; 72 (5): 353-358.

[7] Angelini G, Ketzler J, Coursin D. Perioperative care of the diabetic patient. ASA Refresher Courses in Anesthesiology. 2001; 29 (1): 1-9.

[8] Zacharias A, Habib RH. Factors predisposing to median sternotomy complications: deep vs superficial infection. Chest. 1996; 110 (5): 1173-1178.

[9] Turchin A, Matheny ME, Shubina M, Scanlon JV, Greenwood B, Pendergrass ML. Hypoglycemia and clinical outcomes in patients with diabetes hospitalized in the general ward. Diabetes care. 2009; 32 (7): 1153-1157.

[10] Association AD. Defining and reporting hypoglycemia in diabetes: a report from the American Diabetes Association Workgroup on Hypoglycemia. Diabetes Care. 2005; 28 (5): 1245-1249.

[11] Anthony M. Treatment of hypoglycemia in hospitalized adults. The Diabetes Educator. 2007; 33 (4): 709-715. 
[12] Arias P, Kerner W, Zier H, Navascués I, Pfeiffer EF. Incidence of hypoglycemic episodes in diabetic patients under continuous subcutaneous insulin infusion and intensified conventional insulin treatment: assessment by means of semiambulatory 24-hour continuous blood glucose monitoring. Diabetes Care. 1985; 8 (2): 134-140.

[13] Yue X, Wu J, Ruan Z, Wolden ML, Li L, Lin Y. The Burden of Hypoglycemia in Patients With Insulin-Treated Diabetes Mellitus in China: Analysis of Electronic Medical Records From 4 Tertiary Hospitals. Value in health regional issues. 2020; 21: $17-21$

[14] Quilliam BJ, Simeone JC, Ozbay AB. Risk factors for hypoglycemia-related hospitalization in patients with type 2 diabetes: a nested case-control study. Clinical therapeutics. 2011; 33 (11): 1781-1791.

[15] Zammitt NN, Frier BM. Hypoglycemia in type 2 diabetes: pathophysiology, frequency, and effects of different treatment modalities. Diabetes care. 2005; 28 (12): 2948-2961.

[16] Miller ME, Bonds DE, Gerstein HC, Seaquist ER, Bergenstal RM, Calles-Escandon J, et al. The effects of baseline characteristics, glycaemia treatment approach, and glycated haemoglobin concentration on the risk of severe hypoglycaemia: post hoc epidemiological analysis of the ACCORD study. Bmj. 2010; 340: b5444-5456.
[17] Zoungas S, Patel A, Chalmers J, de Galan BE, Li Q, Billot L, et al. Severe hypoglycemia and risks of vascular events and death. New England Journal of Medicine. 2010; 363 (15): 1410-1418.

[18] Group UHS. Risk of hypoglycaemia in types 1 and 2 diabetes: effects of treatment modalities and their duration. Diabetologia. 2007; 50 (6): 1140-1147.

[19] Koye D, Shaw J, Reid C, Atkins RC, Reutens AT, Magliano DJ. Incidence of chronic kidney disease among people with diabetes: a systematic review of observational studies. Diabetic Medicine. 2017; 34 (7): 887-901.

[20] Liu L, Ke W, Wan X, Zhang P, Cao X, Deng W, et al. Insulin requirement profiles of short-term intensive insulin therapy in patients with newly diagnosed type 2 diabetes and its association with long-term glycemic remission. Diabetes research and clinical practice. 2015; 108 (2): 250-257.

[21] Akram K, Pedersen-Bjergaard U, Carstensen B, Borch-Johnsen K, Thorsteinsson B. Frequency and risk factors of severe hypoglycaemia in insulin-treated Type 2 diabetes: a cross-sectional survey. Diabetic medicine. 2006; 23 (7): 750-756.

[22] Henderson J, Allen K, Deary I, Frier BM. Hypoglycaemia in insulin-treated Type 2 diabetes: frequency, symptoms and impaired awareness. Diabetic Medicine. 2003; 20 (12): 1016-1021. 